\title{
A quantum bound on the compactness
}

\author{
Roberto Casadio $^{1,2, a_{(D)}}$ \\ ${ }^{1}$ Dipartimento di Fisica e Astronomia, Università di Bologna, via Irnerio 46, 40126 Bologna, Italy \\ ${ }^{2}$ I.N.F.N., Sezione di Bologna, I.S. FLAG, viale B. Pichat 6/2, 40127 Bologna, Italy
}

Received: 22 May 2021 / Accepted: 29 December 2021 / Published online: 7 January 2022

(C) The Author(s) 2022

\begin{abstract}
We present a simple quantum description of the gravitational collapse of a ball of dust which excludes those states whose width is arbitrarily smaller than the gravitational radius of the matter source and supports the conclusion that black holes are macroscopic extended objects. We also comment briefly on the relevance of this result for the ultraviolet self-completion of gravity and the connection with the corpuscular picture of black holes.
\end{abstract}

\section{Introduction and motivation}

It has been often argued that quantum gravity should remove the singularity predicted by General Relativity at the endpoint of the gravitational collapse [1]. We study this issue by considering a simple quantum description of the gravitational collapse of a ball of dust [2]. Since gravity is the only interaction acting on dust, the areal radius $R$ of the ball classically follows a radial geodesic in the (outer) Schwarzschild spacetime $^{1}$

$$
\begin{aligned}
\mathrm{d} s^{2}= & -\left(1-\frac{2 G_{\mathrm{N}} M}{r}\right) \mathrm{d} t^{2} \\
& +\left(1-\frac{2 G_{\mathrm{N}} M}{r}\right)^{-1} \mathrm{~d} r^{2} \\
& +r^{2} \mathrm{~d} \Omega^{2},
\end{aligned}
$$

where $M$ is the Arnowitt-Deser-Misner (ADM) [3] mass of the dust. In analogy with the quantum mechanics of the hydrogen atom, in which one quantises the position of the electron with respect to the centre-of-mass of the system, only

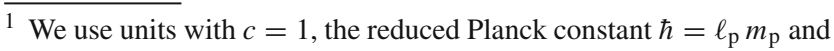
the Newton constant $G_{\mathrm{N}}=\ell_{\mathrm{p}} / m_{\mathrm{p}}$, where $\ell_{\mathrm{p}}$ is the Planck length and $m_{\mathrm{p}}$ the Planck mass.

a e-mail: casadio@bo.infn.it (corresponding author)
}

the radius $R$ of the ball will be quantised here. ${ }^{2}$ The radial geodesic equation will then become a time-independent Schrödinger equation for a particle in the Newtonian potential, with the important feature that quantum states with width significantly smaller than the gravitational radius

$R_{\mathrm{H}}=2 G_{\mathrm{N}} M$

are not physically allowed. This is similar to the quantum removal of the classical UV catastrophe in the hydrogen atom, except that the mass $M$ naturally introduces a lower bound on the energy spectrum which would be absent in the Newtonian approximation. One could then infer that the quantum nature of black holes as extended objects follows from the non-linearity of the gravitational interaction as it is described by General Relativity.

The main results will be obtained in the next section, with more speculative considerations summarised in Sect. 3 .

\section{Minisuperspace model for the gravitational collapse}

We consider a self-gravitating ball of dust of radius $R$ and ADM mass $M[2,10]$. In General Relativity, the surface of the ball follows a radial geodesic in the Schwarzschild spacetime (1.1) and its areal radius $R=R(\tau)$ must therefore satisfy an equation of the form ${ }^{3}$

$$
\left(\frac{\mathrm{d} R}{\mathrm{~d} \tau}\right)^{2}+1-\frac{2 G_{\mathrm{N}} M}{R} \simeq \frac{E^{2}}{M^{2}}
$$

where $\tau$ is the proper time and $E<M$ is the conserved energy of a bound trajectory. The above can be rewritten as

\footnotetext{
${ }^{2}$ For more refined approaches in which the singularity is also removed, see e.g. Refs. [4-9].

${ }^{3}$ Numerical coefficients of order one will often be omitted or approximated for the sake of clarity, given that the simplicity of the model diminishes their physical relevance. A more precise analysis is left for future refinements.
} 
$H \equiv \frac{P^{2}}{2 M}-\frac{G_{\mathrm{N}} M^{2}}{R}=\frac{M}{2}\left(\frac{E^{2}}{M^{2}}-1\right) \equiv \mathcal{E}$,

where we introduced the momentum $P=M(\mathrm{~d} R / \mathrm{d} \tau)$. One can immediately notice that Eq. (2.2) is formally the same as the Newtonian equation for energy conservation. In fact, Eq. (2.2) is the mass-shell condition for the ball and the Hamiltonian constraint of General Relativity for dust.

The study of spherically symmetric gravitational collapse in the quantum theory has a long history (for a partial selection of papers, see Refs. [4,6-9,11-13]). We here proceed with a simple quantisation prescription for the above equation by introducing the momentum operator $\hat{P}=-i \hbar \partial / \partial R$. This is physically equivalent to assuming that the radius of the ball $R$ satisfies an uncertainty relation stemming from the usual canonical commutator, that is

$[\hat{R}, \hat{P}]=i \hbar \Rightarrow \Delta R \Delta P \gtrsim \hbar=\ell_{\mathrm{p}} m_{\mathrm{p}}$,

where $\Delta O \equiv\left\langle\hat{O}^{2}\right\rangle-\langle\hat{O}\rangle^{2}$ for $\hat{O}=\hat{R}$ or $\hat{P}$. Moreover, all of the expectation values are taken on wavefunctions $\Psi=$ $\Psi(R)$ satisfying

$\hat{H} \Psi=\mathcal{E} \Psi$,

which is just the time-independent Schrödinger equation for a gravitational (Newtonian) atom. In particular, the energy spectrum contains the eigenstates

$\Psi_{n} \simeq \sqrt{\frac{M^{9}}{\pi n^{5} \ell_{\mathrm{p}}^{3} m_{\mathrm{p}}{ }^{9}}} e^{-\frac{M^{3} r}{n m_{\mathrm{p}} \ell_{\mathrm{p}}}} L_{n-1}^{1}\left(\frac{2 M^{3} r}{n m_{\mathrm{p}}^{3} \ell_{\mathrm{p}}}\right)$.

where the integer number $n \geq 1$ and $L_{n-1}^{1}$ are the generalised Laguerre polynomials (for zero angular momentum ${ }^{4}$ ). The corresponding eigenvalues are given by

$\frac{\mathcal{E}_{n}}{M} \simeq-\frac{G_{\mathrm{N}}^{2} M^{4}}{2 \hbar^{2} n^{2}}=-\frac{1}{2 n^{2}}\left(\frac{M}{m_{\mathrm{p}}}\right)^{4}=\frac{1}{2}\left(\frac{E_{n}^{2}}{M^{2}}-1\right)$,

and one also has

$R_{n} \equiv\left\langle\Psi_{n}|R| \Psi_{n}\right\rangle \simeq \frac{\hbar^{2} n^{2}}{G_{\mathrm{N}} M^{3}}=n^{2} \ell_{\mathrm{p}}\left(\frac{m_{\mathrm{p}}}{M}\right)^{3}$.

At first sight, it thus appears that the spectrum contains states $\Psi_{n}$ of infinitesimally small width, since $R_{1} \sim$ $\ell_{\mathrm{p}}\left(m_{\mathrm{p}} / M\right)^{3} \ll \ell_{\mathrm{p}}$ for any macroscopic objects, like stars, whose mass $M \gg m_{\mathrm{p}}$. This ground state would have an energy density of the order of $M / R_{1}^{3} \sim\left(M^{10} / m_{\mathrm{p}}{ }^{9}\right) \ell_{\mathrm{p}}^{-3}$, which can hardly be considered a satisfying alternative to the classical singularity of infinite energy density.

\footnotetext{
${ }^{4}$ An obvious generalisation is to consider a spinning ball, but that breaks spherical symmetry, and a treatment fully consistent with General Relativity would thus become much more involved.
}

However, Eq. (2.6) yields

$0 \leq \frac{E_{n}^{2}}{M^{2}} \simeq 1-\frac{1}{n^{2}}\left(\frac{M}{m_{\mathrm{p}}}\right)^{4}$,

tantamount to $\mathcal{E} \gtrsim-M / 2$ in Eq. (2.2), and we thus find that acceptable states $\Psi_{n}$ must satisfy

$n \geq N_{M} \simeq\left(\frac{M}{m_{\mathrm{p}}}\right)^{2}$.

Correspondingly, we obtain

$R_{n} \gtrsim R_{N_{M}} \sim R_{\mathrm{H}}$

which results in the quantum upper bound

$\frac{G_{\mathrm{N}} M}{R_{n}} \lesssim 1$

Moreover, we observe that the Hamiltonian eigenvalues are also bounded from below as (see also Ref. [14])

$\mathcal{E}_{n} \geq \mathcal{E}_{N_{M}} \simeq-\frac{M}{2}$,

which corresponds to

$E_{n}^{2} \geq E_{N_{M}}^{2} \simeq 0$.

Since any semiclassical state representing the collapsing ball must be described in terms of superpositions of the $\Psi_{n}$ 's with $n \geq N_{M}$, the above quantum bound (2.11) implies that any ball of dust must have compactness $G_{\mathrm{N}} M / R \lesssim 1$. It is important to stress once more that this result holds up to a factor of order one and better estimates should be obtained only by considering more realistic and complete models. However, it appears rather unnatural that such refinements can bring down the minimum size from a fraction of $R_{\mathrm{H}}$ to $\ell_{\mathrm{p}}$.

The present minisuperspace description only contains the observable $R$. From the wavefunction $\Psi=\Psi(R)$, we can therefore only determine such information as the expectation value of $R$ and the probability that the ball be inside the gravitational radius,

$$
\begin{aligned}
P\left(R \leq R_{\mathrm{H}}\right) & \equiv \int_{0}^{R_{\mathrm{H}}} \mathcal{P}(R) \mathrm{d} R \\
& =4 \pi \int_{0}^{R_{\mathrm{H}}}|\Psi(R)|^{2} R^{2} \mathrm{~d} R,
\end{aligned}
$$

which can be viewed as the probability that the dust ball is a black hole (when the mass $M$ is treated as a fixed parameter ${ }^{5}$ ). For the ground state, whose wavefunction is given by

$\Psi=\Psi_{N_{M}} \simeq \sqrt{\frac{m_{\mathrm{p}}}{\pi \ell_{\mathrm{p}}^{3} M}} e^{-\frac{M r}{m_{\mathrm{p}} \ell_{\mathrm{p}}}} L_{\frac{M^{2}}{m_{\mathrm{p}}^{2}}-1}^{1}\left(\frac{2 M r}{m_{\mathrm{p}} \ell_{\mathrm{p}}}\right)$,

\footnotetext{
5 Alternative viewpoints are considered in Refs. [15-18], where the mass $M$ is quantised, and in Ref. [19], where $M$ is allowed a statistical uncertainty. We will comment again about the former approach in Sect. 3.
} 

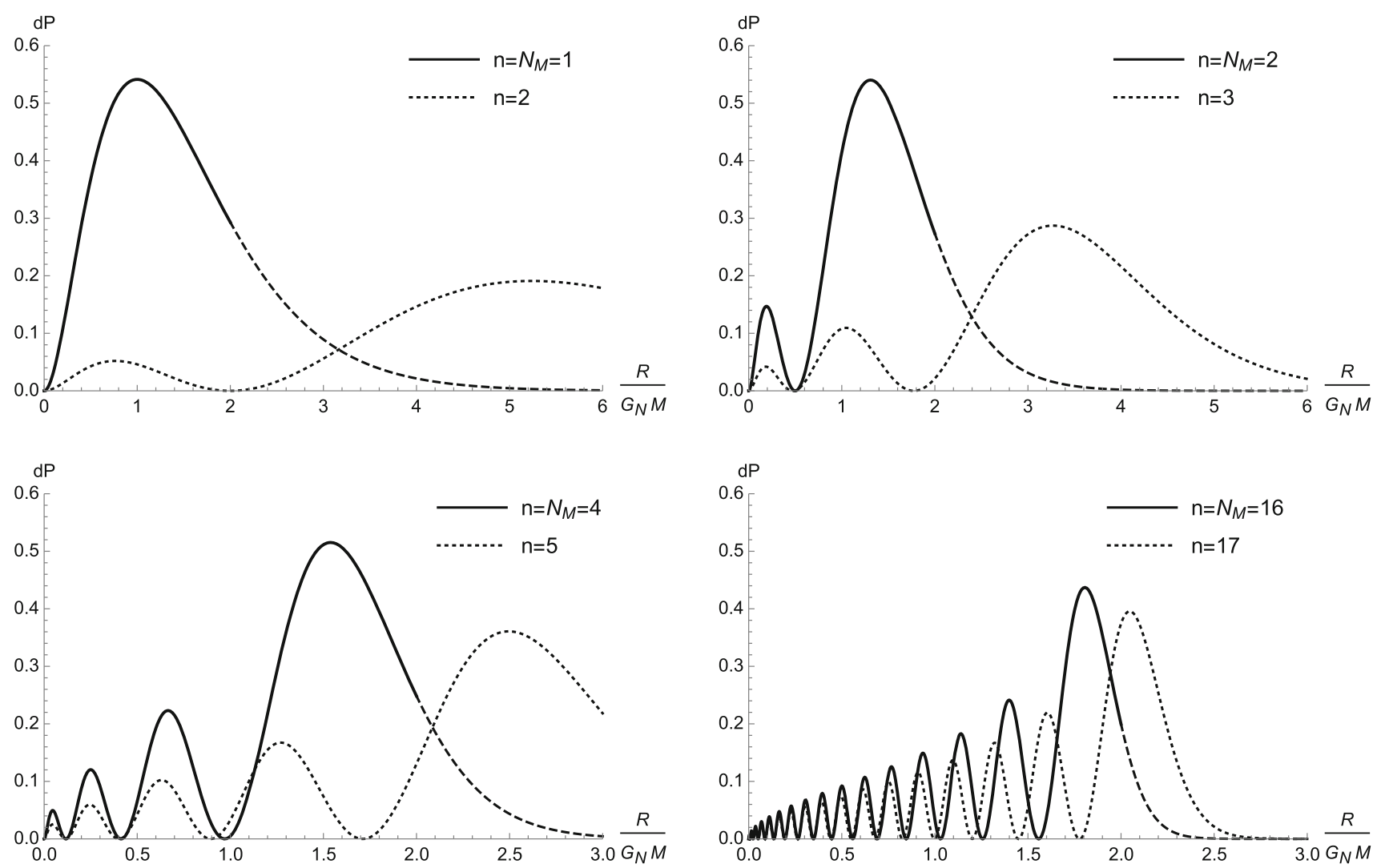

Fig. 1 Probability density $\mathcal{P}=\mathcal{P}(R)$ for: (a) ground state with $n=N_{M}=M^{2} / m_{\mathrm{p}}{ }^{2}$ [solid (dashed) line represents the region inside (outside) the gravitational radius $R_{\mathrm{H}}=2 G_{\mathrm{N}} M$ ]; (b) first excited state with $n=N_{M}+1$ (dotted line)

the probability density $\mathcal{P}=\mathcal{P}_{N_{M}}(R)$ is plotted in Fig. 1 for a few cases with very small $M$. For values of $M \gg m_{\mathrm{p}}$, the probability density narrowly peaks around a value of $R$ slightly below $R_{\mathrm{H}}$ and one thus finds

$P_{N_{M}}\left(R \leq R_{\mathrm{H}}\right) \simeq 1$.

Moreover, the width of this highest peak

$\Delta R_{N_{M}} \sim \frac{R_{N_{M}}}{N_{M}} \sim \ell_{\mathrm{p}} \frac{m_{\mathrm{p}}}{M}$.

As expected, $\Delta R_{N_{M}} \ll R_{N_{M}}$, hence the radius of a very massive matter source should behave like a classical variable.

Figure 1 also displays the first excited state, which asymptotically approaches the corresponding ground state for increasing $M$. This implies that $P_{n}\left(R \leq R_{\mathrm{H}}\right) \simeq 1$ for a range of states $n \gtrsim N_{M}$, which suggests that large astrophysical black holes of a given mass $M$ might not necessarily be in their ground state with $n=N_{M}$. In fact, for $n \gtrsim N_{M}$, the quantum of the Hamiltonian $H$ in Eq. (2.2) is given by

$\delta H \equiv\left|\mathcal{E}_{n+1}-\mathcal{E}_{n}\right| \simeq m_{\mathrm{p}} \frac{m_{\mathrm{p}}}{M}$,

so that $\delta H \sim m_{\mathrm{p}}\left(\Delta R_{N_{M}} / \ell_{\mathrm{p}}\right) \ll m_{\mathrm{p}}$ for a macroscopic object of mass $M \gg m_{\mathrm{p}}$. Furthermore, since

$\delta E \equiv\left|E_{n+1}-E_{n}\right| \simeq m_{\mathrm{p}}$, it appears that the proper source "energy" is naturally quantised in units of the fundamental Planck mass $m_{\mathrm{p}}$, but this quantum is redshifted down to the much smaller $\delta H$ measured by outer observers.

\section{Concluding remarks and outlook}

We have shown how the General Relativistic description of gravity leads to an upper bound for the compactness of a ball of dust in the quantum theory. It is interesting to notice that the lower bounds on the eigenvalue $\mathcal{E}_{n} \geq \mathcal{E}_{N_{M}}$ and radius $R_{n} \gtrsim G_{\mathrm{N}} M$ could not be obtained in the Newtonian theory, since $\mathcal{E}=E$ in that approximation, and the entire spectrum $\Psi_{n}$ with $n \geq 1$ would be physically acceptable therein. The bound (2.11) on the compactness therefore follows from the nonlinearity of General Relativity and agrees with previous results [20] obtained by adding a gravitational self-interaction term to the Newtonian theory [21-24]. It also agrees with those results following from the quantum description of the gravitational radius and black hole horizon [15-18]. In particular, the latter approach leads to very similar conclusions to the ones shown in the previous section, like the black hole probability (2.16) and radius uncer- 
tainty (2.17), when the self-gravitating object is described by an extended many-body system with a very large occupation number of order $N_{M} \sim M^{2} / m_{\mathrm{p}}{ }^{2}$ in its ground state [25]. The first excited modes could then be populated thermally [25] and reproduce the Hawking radiation [26].

It is indeed very intriguing that Eq. (2.9) and the bound (2.11) allow for recovering the fundamental scaling relations employed in the corpuscular description of black holes [27-33]. In fact, the allowed state of minimum energy (2.15) has principal quantum number

$N_{M} \simeq N_{\mathrm{G}}$,

where $N_{\mathrm{G}}$ is the number of soft gravitons in the coherent state representing the gravitational potential generated by a source of mass $M$ [34-37]. We recall that the corpuscular picture naturally reproduces Bekenstein's area spectrum $G_{\mathrm{N}}^{2} M^{2} \sim$ $N_{\mathrm{G}} \ell_{\mathrm{p}}^{2}$ [38], so that the black hole ADM mass is quantised in units of

$\delta M \simeq \delta H$,

where $\delta H$ is precisely the quantum of our Hamiltonian given in Eq. (2.18). This is also the typical energy of particles emitted by the Hawking evaporation process, which the corpuscular picture describes as the depletion of the quantum state of gravity [27-33].

In this perspective, the wavefunction $\Psi_{N_{M}}=\Psi_{N_{\mathrm{G}}}$ appears as the "non-perturbative ground state" for selfgravitating macroscopic objects of mass $M$ and should thus be the closest possible to a classical black hole (as we have already commented at the end of the previous section). This result is consistent with the fact that a static gravitational field must be fully determined by the state of the source, as it does not contain independent degrees of freedom in a static system [15-18]. It can further be interpreted as the fact that the quantum state of a macroscopic self-gravitating system of mass $M$ is very far from the vacuum $\Psi_{0}$ of quantum gravity, for which $N_{\mathrm{G}}=0$. The number $N_{M} \sim N_{\mathrm{G}} \sim M^{2}$ hence provides a quantitative measure for this "distance" from the vacuum in the Hilbert space of quantum gravity states. The appearance of the ground state $\Psi_{N_{M}}=\Psi_{N_{\mathrm{G}}}$ when the ADM energy is given by Eq. (2.9), in turn, can be interpreted as a form of classicalization ensuring the ultraviolet self-completeness of gravity [39-42], because states with spatial momenta much larger than $\hbar / R_{N_{M}} \sim \hbar / R_{\mathrm{H}}$ have $n<N_{M}$ and cannot be populated at energy scales of the order of the mass $M$.

We note that, although quantum states corresponding to the classical singularity appear to be removed from the spectrum, it would be phenomenologically very important to determine a more precise maximum value of the compactness in order to assess the size of quantum deviations from the exterior Schwarzschild geometry. Moreover, in order to make contact with actual observations, one needs an explicit description of the exterior spacetime and of the interaction among the collapsing object and signals that can reach out to our detectors. For example, an effectively finite size of the self-gravitating system is expected to induce the kind of quantum deviations obtained in Ref. [37] from a ultraviolet cut-off for the momenta generating the outer mean-field geometry. A unified description of the quantum states of the collapsing ball presented here and the quantum gravitational potential of Ref. [37] is left for future investigations.

We conclude by remarking that spinning objects and electrically charged sources would also be very interesting to consider. In particular, charged sources have already been analysed by means of the horizon quantum mechanics [1518], for which it was then shown that the inner Cauchy horizon of the classical Reissner-Nordström black hole has vanishing probability to occur [43] and that quantum fluctuations do not allow for the existence of an event horizon if the source is (significantly) overcharged [44]. The case of rotating black holes was likewise analysed in Ref. [45], and the existence of the inner Cauchy horizon of the Kerr geometry was again found to be highly disfavoured, although the angular momentum could be included only as an asymptotic quantity. We expect the present approach will lead to similar conclusions, although more preliminary work is necessary in order to describe the collapse of rotating or electrically charged objects by means of a manageable minisuperspace.

Acknowledgements R.C. is partially supported by the INFN grant FLAG and his work has also been carried out in the framework of activities of the National Group of Mathematical Physics (GNFM, INdAM) and COST action Cantata.

Data Availability Statement This manuscript has no associated data or the data will not be deposited. [Authors' comment: This is a theoretical work which involves no data.]

Open Access This article is licensed under a Creative Commons Attribution 4.0 International License, which permits use, sharing, adaptation, distribution and reproduction in any medium or format, as long as you give appropriate credit to the original author(s) and the source, provide a link to the Creative Commons licence, and indicate if changes were made. The images or other third party material in this article are included in the article's Creative Commons licence, unless indicated otherwise in a credit line to the material. If material is not included in the article's Creative Commons licence and your intended use is not permitted by statutory regulation or exceeds the permitted use, you will need to obtain permission directly from the copyright holder. To view a copy of this licence, visit http://creativecomm ons.org/licenses/by/4.0/.

Funded by $\mathrm{SCOAP}^{3}$.

\section{References}

1. S.W. Hawking, G.F.R. Ellis, The Large Scale Structure of SpaceTime (Cambridge University Press, Cambridge, 1973)

2. J.R. Oppenheimer, H. Snyder, Phys. Rev. 56, 455 (1939)

3. R.L. Arnowitt, S. Deser, C.W. Misner, Phys. Rev. 116, 1322 (1959)

4. V.P. Frolov, G.A. Vilkovisky, Phys. Lett. B 106, 307 (1981) 
5. D. Malafarina, Universe 3, 48 (2017). arXiv:1703.04138 [gr-qc]

6. R. Casadio, Int. J. Mod. Phys. D 9, 511 (2000). arXiv:gr-qc/9810073

7. H.M. Haggard, C. Rovelli, Phys. Rev. D 92, 104020 (2015). arXiv:1407.0989 [gr-qc]

8. W. Piechocki, T. Schmitz, Phys. Rev. D 102, 046004 (2020). arXiv:2004.02939 [gr-qc]

9. T. Schmitz, Phys. Rev. D 103, 064074 (2021). arXiv:2012.04383 [gr-qc]

10. H. Stephani, Relativity (Cambridge University Press, Cambridge, 2004)

11. P. Hajicek, B.S. Kay, K.V. Kuchar, Phys. Rev. D 46, 5439 (1992)

12. P. Hajicek, C. Kiefer, Int. J. Mod. Phys. D 10, 775 (2001). arXiv:gr-qc/0107102 [gr-qc]

13. R. Casadio, G. Venturi, Class. Quantum Gravity 13, 2715 (1996). arXiv:gr-qc/9512032

14. N. Dadhich, JCAP 04, 035 (2020). arXiv:1903.03436 [gr-qc]

15. R. Casadio, Localised particles and fuzzy horizons: a tool for probing quantum black holes. arXiv:1305.3195 [gr-qc]

16. R. Casadio, F. Scardigli, Eur. Phys. J. C 74, 2685 (2014). arXiv: 1306.5298 [gr-qc]

17. R. Casadio, A. Giugno, O. Micu, A. Orlandi, Phys. Rev. D 90, 084040 (2014). arXiv:1405.4192 [hep-th]

18. R. Casadio, A. Giugno, A. Giusti, Gen. Relativ. Gravit. 49, 32 (2017). arXiv:1605.06617 [gr-qc]

19. R. Casadio, A. Giusti, A. Mentrelli, Phys. Rev. D 100, 024036 (2019). arXiv:1901.06206 [gr-qc]

20. R. Casadio, M. Lenzi, A. Ciarfella, Phys. Rev. D 101, 124032 (2020). arXiv:2002.00221 [gr-qc]

21. R. Casadio, M. Lenzi, O. Micu, Phys. Rev. D 98, 104016 (2018). arXiv: 1806.07639 [gr-qc]

22. R. Casadio, M. Lenzi, O. Micu, Eur. Phys. J. C 79, 894 (2019). arXiv: 1904.06752 [gr-qc]

23. R. Casadio, I. Kuntz, Eur. Phys. J. C 80, 581 (2020). arXiv:2003.03579 [gr-qc]

24. R. Casadio, A. Giusti, I. Kuntz, G. Neri, Phys. Rev. D 103, 064001 (2021). arXiv:2101.12471 [gr-qc]

25. R. Casadio, A. Giugno, A. Orlandi, Phys. Rev. D 91, 124069 (2015). arXiv:1504.05356 [gr-qc]
26. S.W. Hawking, Commun. Math. Phys. 43, 199 (1975) [Erratum: Commun. Math. Phys. 46, 206 (1976)]

27. G. Dvali, C. Gomez, Fortsch. Phys. 61, 742 (2013). arXiv:1112.3359 [hep-th]

28. G. Dvali, C. Gomez, S. Mukhanov, Black hole masses are quantized. arXiv:1106.5894 [hep-ph]

29. G. Dvali, C. Gomez, Phys. Lett. B 719, 419 (2013). arXiv:1203.6575 [hep-th]

30. G. Dvali, C. Gomez, Phys. Lett. B 716, 240 (2012). arXiv:1203.3372 [hep-th]

31. G. Dvali, C. Gomez, Eur. Phys. J. C 74, 2752 (2014). arXiv:1207.4059 [hep-th]

32. S. Hofmann, T. Rug, Nucl. Phys. B 902, 302 (2016). arXiv:1403.3224 [hep-th]

33. A. Giusti, Int. J. Geom. Meth. Mod. Phys. 16, 1930001 (2019)

34. R. Casadio, A. Giugno, A. Giusti, Phys. Lett. B 763, 337 (2016). arXiv:1606.04744 [gr-qc]

35. W. Mück, Can. J. Phys. 92, 973 (2014). arXiv:1306.6245 [hep-th]

36. R. Casadio, A. Giugno, A. Giusti, M. Lenzi, Phys. Rev. D 96, 044010 (2017). arXiv: 1702.05918 [gr-qc]

37. R. Casadio, Quantum black holes and resolution of the singularity. arXiv:2103.00183 [gr-qc]

38. J.D. Bekenstein, Phys. Rev. D 7, 2333 (1973)

39. G. Dvali, G.F. Giudice, C. Gomez, A. Kehagias, JHEP 1108, 108 (2011). arXiv:1010.1415 [hep-ph]

40. G. Dvali, D. Pirtskhalava, Phys. Lett. B 699, 78 (2011). arXiv:1011.0114 [hep-ph]

41. G. Dvali, C. Gomez, A. Kehagias, JHEP 1111, 070 (2011). arXiv:1103.5963 [hep-th]

42. R. Percacci, L. Rachwal, Phys. Lett. B 711, 184 (2012). arXiv:1202.1101 [hep-th]

43. R. Casadio, O. Micu, D. Stojkovic, JHEP 05, 096 (2015). arXiv: 1503.01888 [gr-qc]

44. R. Casadio, O. Micu, D. Stojkovic, Phys. Lett. B 747, 68 (2015). arXiv: 1503.02858 [gr-qc]

45. R. Casadio, A. Giugno, A. Giusti, O. Micu, Eur. Phys. J. C 77, 322 (2017). arXiv:1701.05778 [gr-qc] 